\title{
Management of the displaced intra articular fracture calcaneum by open reduction \& internal fixation with the locking calcaneum plate: A study
}

\author{
Yogesh Sisodia ${ }^{1, *}$, Mukesh Haritwal ${ }^{2}$, Rajesh Goel ${ }^{3}$, Kishan Gopal Nama ${ }^{4}$ \\ ${ }^{\mathbf{1 , 4}}$ Assistant Professor, ${ }^{2}$ Senior Resident, ${ }^{3}$ Professor \& HOU, Dept. of Orthopaedics, ${ }^{1}$ Bharati Vidyapeeth Deemed University \\ Medical College, Pune, Maharashtra, ${ }^{2}$ Sir Gangaram Hospital, New Delhi, Delhi, ${ }^{3}$ New Medical College, Kota, Rajasthan, \\ ${ }^{4}$ Maharao Bhimsingh Hospital, Kota, Rajasthan, India
}

*Corresponding Author:

Email: yogeshsportsdoc@gmail.com

\begin{abstract}
Introduction: Since the mid-nineties, open reduction and internal fixation is considered as the gold standard treatment for the displaced intra-articular fractures of the calcaneus by most experts. However, there still remains dilemma regarding the adequate management strategy for these complex and unsolved fractures \& hence, the intent of our study is to resolve many of the issues related to this fracture.

Materials and Methods: 25 patients of closed fracture calcaneum (Type 2, 3 \& 4 Sanders classification) operated with the open reduction \& internal fixation between the year 2011 and 2014 were included in the study. During follow-up, pain, functional outcome, range of motion etc. were evaluated with the use of the Maryland Foot Score (MFS).

Results: We studied 25 patients who had a combined total of 30 closed displaced intra-articular calcaneal fractures of Type 2, 3 \& 4 (Sanders classification) for a mean follow-up period of 13.4+/-1.35 months. Complications seen post-operatively were persistent swelling in 2 cases $(6.6 \%)$ \& Skin complications in 2 cases $(6.6 \%)$. The pre and postoperative values of Bohler's angle, Gissane's angle, height and width show restoration of normal anatomy and this change in the values was significant as pvalue was $<0.05$. Maryland foot score system was used to assess functional outcome and average Maryland foot score found to be $79.7+/-12.5$ (Good).
\end{abstract}

Conclusion: Overall quality of fracture reduction, as judged by restoration of Bohler's angle, Gissane's angle, height and width were good. So, open reduction and internal fixation should be the treatment of choice in such fractures.

Level of incidence: Therapeutic Level IV.

Keywords: Calcaneum, Bohler's angle, Gissane's angle, Maryland foot score, Locking calcaneal plate.

\section{Introduction}

Calcaneum fractures are one of the most disabling fractures in human with frequent occurrence during the wage-earning period of life. ${ }^{1}$ Calcaneal fractures are estimated to comprise approximately 1 to $2 \%$ of all fractures. ${ }^{1}$ In all the calcaneum fractures, posterior subtalar joint involvement was approximately $70 \%$. Also, the males were involved more (80\%) as compared to females. There is a considerable amount of literature on calcaneal fractures and their treatment; however the best management approach has yet to be determined. Conservative treatment is generally applicable in undisplaced fractures, compromised soft-tissues and patients with diabetes, peripheral vascular disease, low anticipated compliance or substance abuse. Fracture calcaneum leads to incongruency of the subtatar joint, widening of heel and loss of talo-navicular lever arm, resulting into subtalar arthritis as a complication. ORIF is still considered as a gold standard treatment for the displaced intra-articular fractures of the calcaneum because it restores the subtalar joint and gives an excellent result. ${ }^{1}$ However, there still remains dilemma regarding the adequate management of these complex fractures \& hence, intent of our study was to resolve many of the issues related to this fractures.

\section{Materials and Methods}

This prospective study was composed of 25 fresh cases of closed fracture calcaneum (less than 3 weeks old) of age less than 50 years $\&$ both the sexes between the years 2011-14. Patients with fracture Type 2, 3, \& 4 according to Sanders classification were included in the study. These cases were treated by Open reduction \& internal fixation via a lateral approach. ${ }^{2}$ After a followup period of 1 year, all patients underwent a physical examination and were evaluated in terms of functional outcome. Data regarding complications that were related to the fractured calcaneum were collected and patients were reassessed during follow-up visits.

\section{Radiographic Assessment}

Pre-operative standard radiographs and computed tomography (CT) scans were done routinely for all patients and postoperative standard radiographs were advised routinely to assess the reduction. With the use of preoperative CT scans, the fractures were classified according to the systems of Sanders. ${ }^{3}$ Preoperative and postoperative measurements of the Bohler's angle, ${ }^{4}$ Gissane's angle, ${ }^{5}$ height and width of calcaneum were obtained and compared. 


\section{Surgical Procedure}

The patient was put under general or spinal anaesthesia and placed in a lateral position. Tourniquet applied painting and draping of the parts done under aseptic conditions. A large L shaped surgical incision was made beginning approximately $4 \mathrm{~cm}$ above the tip of the lateral malleolus, midway between the posterior border of the fibula and the Achilles tendon [Fig. 1]. Then the incision was extended proximally, curved below the sural nerve, and then moved upward to the calcaneocuboid joint.

The incision was made down to the bone with the skin, subcutaneous layer and the periosteum kept as a single thick flap. The lateral flap was developed anteriorly to expose the posterior subtalar joint. Then the flap was elevated along with the sural nerve and peroneal tendons. $\mathrm{K}$ wires were then inserted into the lateral malleolus, neck of talus, cuboid and bent further to keep the flap retracted. It provided us with a better exposure and lesser damage to soft tissue. The subtalar joint was opened and the fractures of the lateral calcaneal wall (whenever present) were dissected, in order to expose the fractured and depressed articular fragments.

The reduction manoeurve usually began at the posterior articular surface and further proceeded to the Gissane's angle and body of the calcaneum. However, if varus tilt of the calcaneus prevents anatomic reduction of the posterior facet, the alignment of the body may need to be corrected prior to the reduction of the joint surface.

The fractured lateral wall (whenever present) of the calcaneum was gently opened, leaving the fracture fragments within their periosteal envelope [Fig. 2]. The depressed posterior facet was elevated, with the help of a schanz screw drilled in the posterolateral part of calcaneal tuberosity [Fig. 3]. The articular surface was reduced, lateral wall reposited, and fixation was made using temporary Kirschner wires. Most of the time, posterior facet was restored first followed by medial facet in relation to the sustentaculum tali. The anterior facet and posterior tuberosity were fixed in the last. The reduction was appreciated under direct vision whenever possible.

The Calcaneal plate was then positioned at the appropriate location on the lateral calcaneal wall. The size that best fits the calcaneal anatomy was chosen from small, medium or large one. Then the plate was moulded and contoured to the lateral aspect of the anterior process, posterior facet and to the tuberosity.

The sleeves were first screwed in the hole located on the anterior extremity and in the 2 holes on the posterior extremity of the plate. They allowed axial drilling and perfect insertion of the locking screws. The holes for fixation screws were drilled using the $2.2 \mathrm{~mm}$ drill through the drilling sleeves. [Fig.4] Screw placement was cheked under image intensifier. [Fig. 5]
After putting drain, the flap was opposed using Vicryl 1-0 in subcutaneous plane and skin by Ethilon 20. [Fig. 6] Bulky compression dressing and below knee slab applied.

\section{Postoperative details}

The drain removal was done at 48 hours on the first inspection. Suture and slab removal was done at 3 weeks. Further, non-weight bearing exercises and walking started. Partial weight bearing started 8-10 weeks post-operatively depending on the radiological progress of union and gradually increased to full weight bearing.

\section{Physical Examination}

Physical examination included a thorough assessment of alignment and stability. The range of motion of the ankle and subtalar joint was measured with a goniometer. Following normative values were used for comparison: plantar flexion $\left(15-50^{\circ}\right)$, dorsiflexion $\left(0-25^{\circ}\right)$, inversion $\left(0-40^{\circ}\right)$ \& eversion $(0-$ $\left.20^{\circ}\right) .6,7$

\section{Functional Outcome Measurements}

To quantify pain and functional disability, the Maryland Foot Score (MFS) was used. ${ }^{8}$ The Maryland Foot Score (MFS) consists of subjective and objective variables classified into three major categories: pain, function and functional activities on a scale ranging from 0 to 100 , lower scores indicating greater impairment. MFS: a score of 90 to 100 was graded as excellent, 75 to 89 as good, 50 to 74 as fair and less than 50 points as poor.

\section{Statistical Methods}

The Pre-operative \& post-operative values of Bohler's angle, Gissane's angle, height \& width were compared by paired $\mathrm{T}$ test. The minimum level of significance was fixed at $95 \%$ confidence limit and a pvalue of $<0.05$ was considered significant.

\section{Results}

The present study consists of 30 fractures of calcaneum in 25 patients, treated in the Department of Orthopaedic Surgery, Government Medical College and associated Hospitals, Kota during the year 2011 to 2014. Duration of follow up was 12 months (minimum) to 16 months (maximum) with an average of $13.4+/$ 1.35 months. In the present study there were $10(33.3 \%)$ Type 2 fractures, $14(46.6 \%)$ Type 3 fractures \& 6 (20 $\%)$ Type 4 fractures according to Sander's classification.

20 of 25 patients $(80 \%)$ belonged to $3^{\text {rd }}$ and $4^{\text {th }}$ decade thus indicating the role of dynamic mobility and activity of young male patient making them prone to injury. The average age in the series was 34.2 years. While male to female ratio was $11.5: 1$. In this present series the calcaneal fracture was found to be most 
common in labourers (24\%), showing considerable economic importance of calcaneal fractures in industrial workers. Right side involvement was $48 \%$ as compared to the left side $(32 \%), 5$ patients $(20 \%)$ had bilateral involvement in this study group. Fall from height found to be most common mode of injury (88\%), signifying the role of axial loading due to high velocity impact to the heel during fall from height. 9 patients (36\%) were associated with other bony injuries, among them fracture of spine (12\%) found to be commonest associated injury. Majority of the patients $(56 \%)$ reported within a week time.

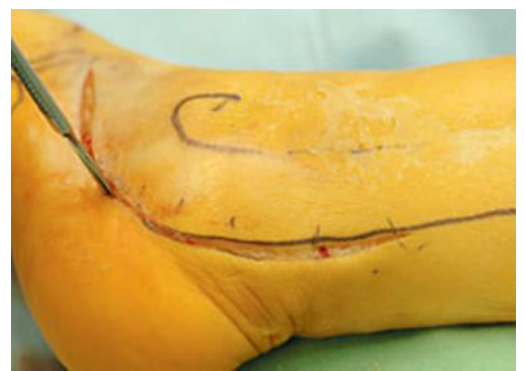

Fig. 1: L shaped skin incision over the lateral aspect of foot

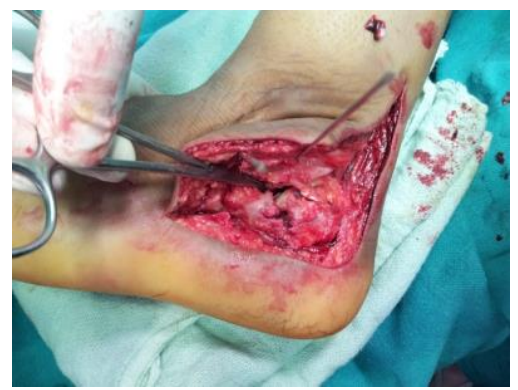

Fig. 2: Exposure of subtalar joint

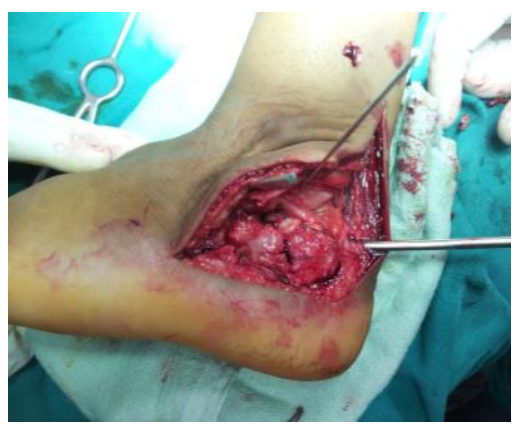

Fig. 3: Reduction of subtalar joint and temporary fixation.

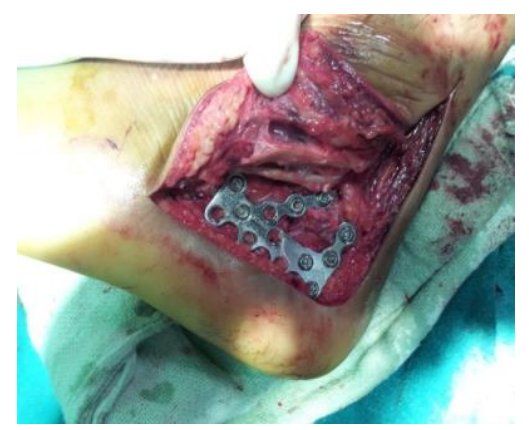

Fig. 4: Fixation with locking calcaneum plate

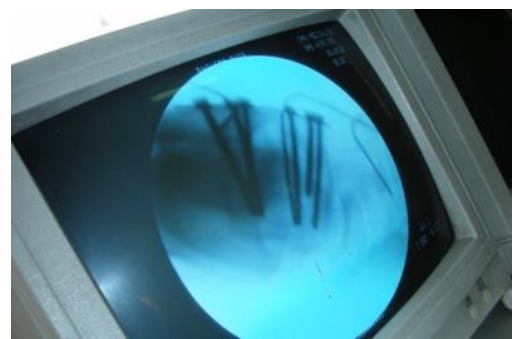

Fig. 5: Fixation seen under image intensifier

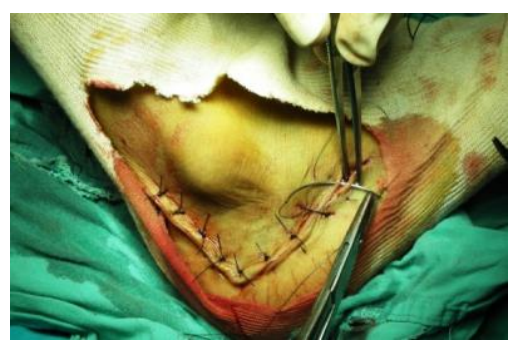

Fig. 6: Closure of incision with non-absorbable sutures

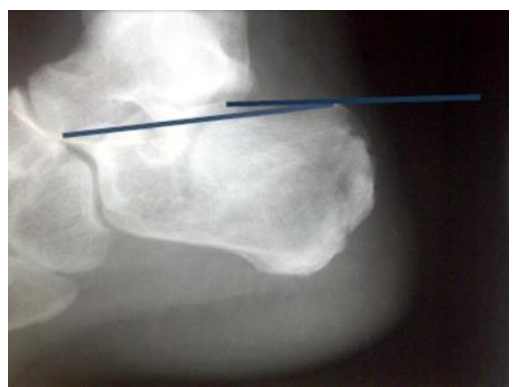

Fig. 7: Pre-operative radiograph showing displaced intra-articular fracture calcaneum

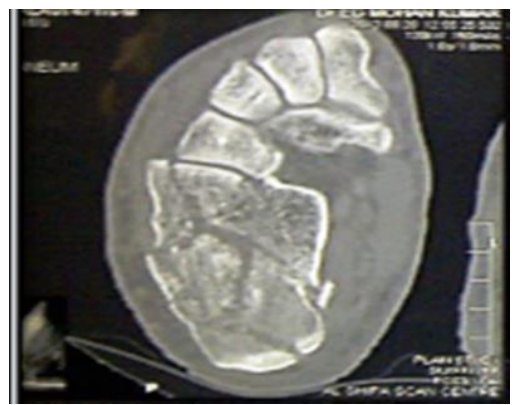

Fig. 8: Pre-operative CT showing displaced intraarticular fracture calcaneum 


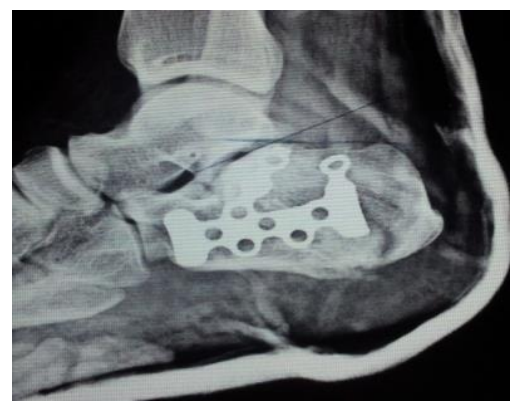

Fig. 9: Post-operative radiograph (Lateral view) showing reduction of subtalar joint

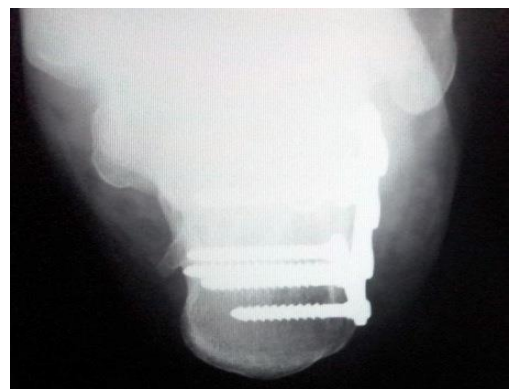

Fig. 10: Post-operative radiograph (Axial view)

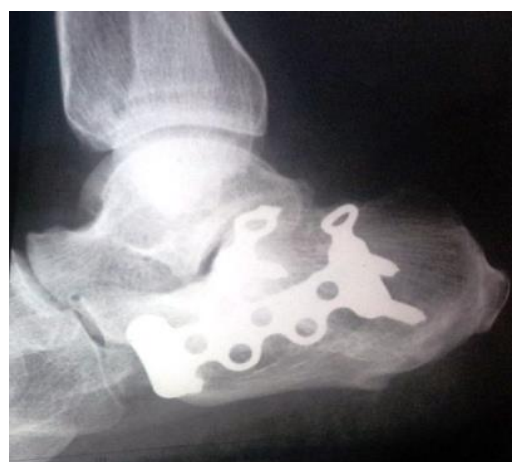

Fig. 11: Post-operative radiograph (Lateral view) at 1 year follow up

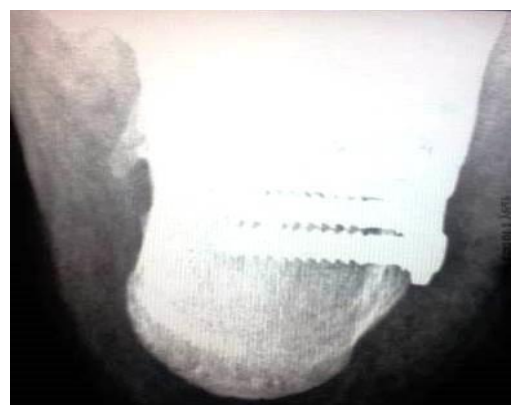

Fig. 12: Post-operative radiograph (Axial view) at 1 year follow up

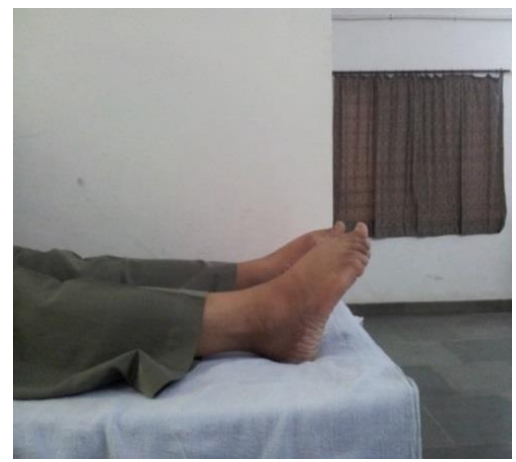

Fig. 13: Range of plantar flexion at the end of 1 year follow up

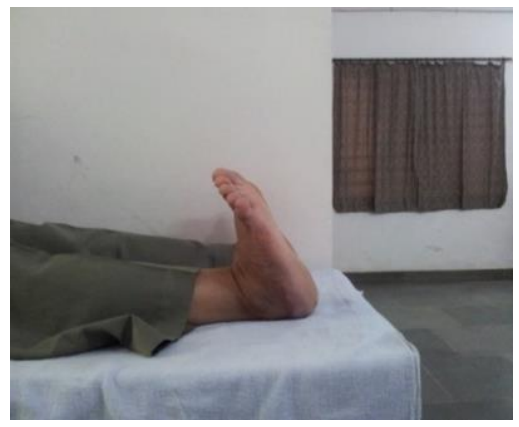

Fig. 14: Range of dorsiflexion at the end of 1 year follow up

\section{Complications}

Complications seen post-operatively were persistent swelling in 2 cases $(6.6 \%)$ \& Skin complication (edge necrosis) in 2 cases $(6.6 \%)$. Majority of cases $(86.6 \%)$ were not associated with any complication. 2 cases showed edge necrosis, although wound healed after serial dressings.

\section{Radiographic examination}

We were able to achieve a mean Bohler's angle of $29.5^{\circ}+/-4.07$ from $15.16^{\circ}+/-5.58$, mean Gissane's angle of $123^{\circ}+/-11.75$ from $160.9^{\circ}+/-7.95$, mean height of $4.17+/-0.27 \mathrm{cms}$ from $3.58+/-0.3 \mathrm{cms} \&$ mean width of $3.7+/-0.18 \mathrm{cms}$ from $4.3+/-0.17 \mathrm{cms}$ by open reduction and internal fixation method. [Table 1] The pre and post-operative comparative values of Bohler's angle, Gissane's angle, height and width were compared using paired ' $\mathrm{T}$ ' test and change in the values found to be significant as $\mathrm{P}$ - value was $<0.05$.

\section{Physical examination}

The passive ranges of motion of the ankle joint and subtalar joint of the treated foot were measured. Mean plantar flexion found to be $33.27+/-8.4$ (Normal range $15-50^{\circ}$ ), mean dorsiflexion $12.7+/-5.4^{\circ}$ (Normal range $0-25^{\circ}$ ), mean inversion $23.26+/-8.19^{\circ}$ (Normal range $\left.0-40^{\circ}\right)$ \& mean eversion found to be $11.53+/-4^{\circ}$ (Normal range $\left.0-20^{\circ}\right)$. [Table 2] 
Functional Outcome Assessments

Maryland foot scoring system was used to assess functional outcome. It measured variables like Pain, Gait, Stability, Support, Limp, Cosmosis, Functional Activities \& Subtalar Motion. A total score was calculated by addition of all individual ratings. Average Maryland foot score found to be 79.7+/- 12.5 (Good). [Table 3]

Excellent results were seen in $23.3 \%$ of cases, Good results in $50 \%$ of cases, Fair results in $20 \%$ of cases \& poor results in $6.6 \%$ of cases. So, in the majority of cases $(50 \%)$ results were good. [Table 4] Persistent swelling was present in 2 cases (6.6\%). Mean interval between injury \& weight bearing in all 25 of cases found to be $88.48+/-23.6$ days.

With the anatomical restoration of the articular surface, the subtalar motion was improved considerably.

\section{Statistical analysis}

Using the paired T-test, pre-operative and postoperative values of Bohler's angle, Gissane's angle, height and width were compared and the change in the values found to be significant as $\mathrm{p}$-value was $<0.0001$.

\section{Discussion}

The treatment of displaced intra-articular fracture of the calcaneus remains controversial. Historically, calcaneal fractures were treated non-operatively with unsatisfactory results. ${ }^{9}$ Due to the improvement of imaging technology and fracture care, surgical procedures have become more popular. ${ }^{10,11}$ Now a days, open reduction and internal fixation is the treatment of choice for displaced intra-articular calcaneal fractures in many institutions. In a prospective, randomized controlled trial, Buckley et al. ${ }^{12}$ demonstrated that operative treatment as a whole provides no improvement over non-operative treatment; however, stratification of the patient population supported the use of surgical care for displaced intra-articular calcaneal fractures. From a meta-analysis, Randle et al ${ }^{13}$ reported a trend for surgically treated patients to have better outcomes but none of the differences reached significance.

Wound complications associated with this fracture are the major concern for many surgeons. Folk et al. ${ }^{14}$ reported a complication rate of $25 \%$ in 190 fractures. Zwipp et al. ${ }^{15}$ reported superficial wound edge necrosis in $8.3 \%$ of their cases. Buckley et al. ${ }^{12}$ showed a superficial wound complication rate of $17 \%$ and a deep infection rate of $5 \%$ in the operative group treated with open reduction and plate fixation. Kundel et $\mathrm{al}^{16}$ reported superficial wound complications in $7 \%$ of their patients, deep infections in 7\%, and a total complication rate of $30 \%$ in the group with open reduction and plate fixation. In our study, minimal complications were found. Skin complications were found in 2 patients (6.6\%) which healed after dressings and persistent swelling in 2 patients $(6.6 \%)$. Decreased rate of skin complications was most probably attributed to the technique of developing thick lateral skin flap.

In our series, we emphasized on the restoration of not only subtalar joint but a realignment of the overall shape of the calcaneum in terms of Bohler's angle, Gissane angle, height and width of the calcaneum. We were able to achieve a mean Bohler's angle of $29.5^{\circ}+/-$ 4.07, mean Gissane's angle of $123^{\circ}+/-11.75$, mean height of $4.17+/-0.27 \mathrm{cms} \&$ mean width of $3.7+/$ $0.18 \mathrm{cms}$ by open reduction and internal fixation method. There was no late collapse noted on final follow up. Leung et $\mathrm{a}^{11}$ using lateral approach reported an average postoperative Bohler's angle of $28.9 \pm 5.9^{0}$, Gissane's angle of $120.10 \pm 5.8^{0}$, height of $4.7 \pm 0.29$ $\mathrm{cms}$ and width of $3.4 \pm 0.3 \mathrm{cms}$.

In the study by Rammelt $\mathrm{S}$ et al ${ }^{17}$ on 18 months of follow-up, mean Maryland foot score was 80.8. In case of our study, it was $79.7+/-12.5$ (Good). In our present study, the overall quality of fracture reduction, as judged by restoration of calcaneal length, height, and width was good.

At the end of the study, we were able to resolve many issues related to management of such fractures. Congruency of subtalar joint was restored by ORIF by inspecting the articular surface of the posterior facet with the naked eye, before applying locking calcaneal plate. Perfect planning of reduction was done by assessing CT scans, to correct displacement of posterior facet fragment and to restore Gissane's angle, Bohler's angle, height and width. Because of congruency of subtalar joint, subtalar and ankle movements were restored to normal range and led to pain-free movements. Thus, patients exhibited normal gait without aids and all of them were able to return to the original profession.

\section{Conclusion}

We have obtained excellent results in $23.3 \%$ of cases \& good result in $50 \%$ cases by open reduction and internal fixation method. Rather the results of this study, in dealing with these difficult types of fractures by open reduction and internal fixation method has encouraged us to treat the calcaneal fractures preferably by this method.

Table 1: Comparative pre and post-operative measurements

\begin{tabular}{|l|c|c|c|}
\hline Characteristic & Pre-operative & Post-operative & P-value \\
\hline Bohler's angle & $15.16+/-5.58$ & $29.5+/-4.07$ & $<0.0001(\mathrm{~S})$ \\
\hline Gissane's angle & $160.9+/-7.95$ & $123+/-11.75$ & $<0.0001(\mathrm{~S})$ \\
\hline Height & $3.58+/-0.3$ & $4.17+/-0.27$ & $<0.0001(\mathrm{~S})$ \\
\hline
\end{tabular}




\begin{tabular}{|l|l|l|l|}
\hline Width & $4.3+/-0.17$ & $3.7+/-0.18$ & $<0.0001$ (S) \\
\hline
\end{tabular}

Table 2: Post-operative assessment of range of motion

\begin{tabular}{|l|c|c|c|c|c|}
\hline $\begin{array}{c}\text { Fracture type } \\
\text { (Sanders } \\
\text { classification) }\end{array}$ & $\begin{array}{c}\text { No. of } \\
\text { fractures }\end{array}$ & $\begin{array}{c}\text { Plantar flexion } \\
\text { (Mean) }\end{array}$ & $\begin{array}{c}\text { Dorsiflexion } \\
\text { (Mean) }\end{array}$ & $\begin{array}{c}\text { Inversion } \\
\text { (Mean) }\end{array}$ & $\begin{array}{c}\text { Eversion } \\
\text { (Mean) }\end{array}$ \\
\hline Type 2 & 10 & $40.6+/-6.93$ & $17.6+/-5$ & $30+/-7.5$ & $14.8+/-3.4$ \\
\hline Type 3 & 14 & $31.86+/-5.5$ & $11.57+/-3.29$ & $21.85+/-5.6$ & $11.28+/-2.7$ \\
\hline Type 4 & 6 & $23.75+/-5.9$ & $7.16+/-3$ & $15.33+/-5.7$ & $6.66+/-2.58$ \\
\hline Overall & 30 & $33.27+/-8.4$ & $12.7+/-5.4$ & $23.26+/-8.19$ & $11.53+/-4$ \\
\hline
\end{tabular}

Table 3: Maryland foot score in various fracture types

\begin{tabular}{|l|c|c|c|c|}
\hline $\begin{array}{c}\text { Fracture type } \\
\text { (Sander's } \\
\text { classification) }\end{array}$ & $\begin{array}{c}\text { No. of } \\
\text { fractures }\end{array}$ & $\begin{array}{c}\text { Maryland } \\
\text { foot score } \\
\text { (Mean) }\end{array}$ & Grade & $\%$ \\
\hline Type 2 & 10 & $87.9+/-9$ & Good & 33.3 \\
\hline Type 3 & 14 & $79.5+/-10.6$ & Good & 46.6 \\
\hline Type 4 & 6 & $65.3+/-10.4$ & Fair & 20 \\
\hline Overall & 30 & $79.7+/-12.5$ & Good & 100 \\
\hline
\end{tabular}

Table 4: Functional outcome assessment using Maryland foot score

\begin{tabular}{|l|c|c|}
\hline \multicolumn{1}{|c|}{ Grade } & No of fractures & \% \\
\hline Excellent & 7 & 23.3 \\
\hline Good & 15 & 50 \\
\hline Fair & 6 & 20 \\
\hline Poor & 2 & 6.6 \\
\hline
\end{tabular}

Graph 1: Post-operative assessment of range of motion at 1 year follow up

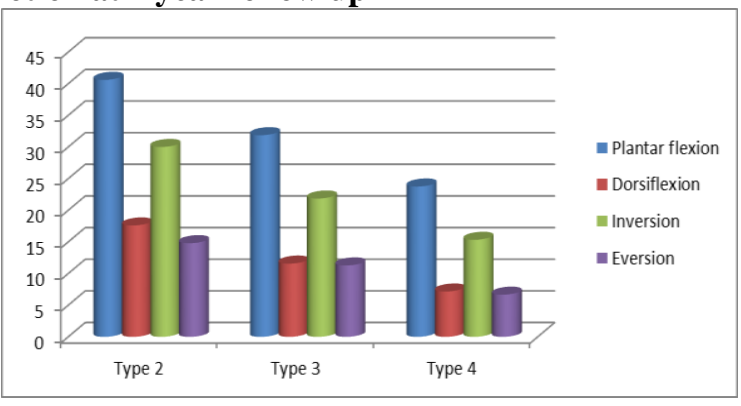

Graph 2: Maryland foot score at 1 year follow up

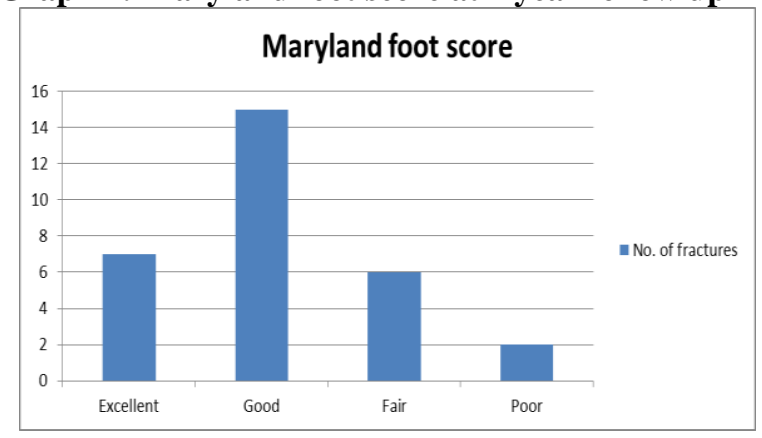

\section{References}

1. Sanders R: Displaced intra-articular fractures of the calcaneus. J Bone Joint Surg Am 2000;82:225-250.

2. Benirschke SK, Sangeorzan BJ. 1993. Extensive intraarticular fractures of the foot. Surgical management of calcaneal fractures. Clinical orthopaedics and related research 292:128-34.

3. Sanders R. Intra-articular fractures of the calcaneus: present state of the art. J Orthop Trauma. 1992;6:252-65.

4. Bohler L. Diagnosis, pathology, and treatment of fractures of the oscalcis. J Bone Joint Surg Am. 1931; 13:75-89.

5. Gissane W. Proceedings of the British Orthopaedic Association. J Bone Joint Surg 1947;29:254-255.

6. Sarrafian SK. Biomechanics of the subtalar joint complex. Clinical orthopaedics and related research 1993;290:17-26.

7. Ryf C, Weymann A. The neutral zero method-a principle of measuring joint function. Injury. 1995;26 (Suppl 1):1-11.

8. Clinical outcome scoring of intra-articular calcaneal fracture. Schepers T, Heetveld MJ, Mulder PG, Patka P. J Foot Ankle Surg. 2008 May-Jun;47(3:213-8)

9. James ET, Hunter GA. The dilemma of painful old oscalcis fractures. Clinical orthopaedics and related research. 1983;177:112-5.

10. Crosby LA, Fitzgibbons T. Computerized tomography scanning of acute intraarticular fractures of the calcaneus. A new classification system. J Bone Joint Surg Am. 1990;72:852-9.

11. Leung KS, Yuen KM, Chan WS. Operative treatment of displaced intra-articular fractures of the calcaneum. Medium-term results. J Bone Joint Surg Br. 1993;75:196201.

12. Buckley R, Tough S, McCormack R, Pate G, Leighton R, Petrie D, Galpin R. Operative compared with nonoperative treatment of displaced intra-articular calcaneal fractures: a prospective, randomized, controlled multicenter trial. J Bone Joint Surg Am. 2002;84:173344.

13. Randle JA, Kreder HJ, Stephen D, Williams J, Jaglal S, Hu R. Should calcaneal fractures be treated surgically? A meta-analysis. Clinical orthopaedics and related research. 2000;377:217-27.

14. Folk JW, Starr AJ, Early JS. Early wound complications of operative treatment of calcaneus fractures: analysis of 190 fractures. J Orthop Trauma. 1999;13:369-72.

15. Zwipp H, Tscherne H, Thermann H, Weber T. Osteosynthesis of displaced intra-articular fractures of the 
calcaneus. Results in 123 cases. Clinical orthopaedics and related research.1993; 290:76-86.

16. Kundel K, Funk E, Brutscher M, Bickel R. Calcaneal fractures: operative versus non-operative treatment. J Trauma. 1996;41:839-45.

17. Rammelt S, Barthel S, Biewener A, Gavlik JM, Zwipp H. Calcaneus fractures. Open reduction and internal fixation Zentralbl Chir. 2003 Jun;128(6):517-28. 